\title{
Inhibition of Protein Phosphatase 2A Sensitizes Mucoepidermoid Carcinoma to Chemotherapy via the PI3K-AKT Pathway in Response to Insulin Stimulus
}

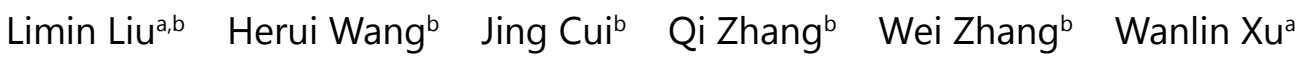 \\ Hao Lu ${ }^{a}$ Shengwen Liu ${ }^{a}$ Shukun Shen ${ }^{a}$ Francia Fang ${ }^{c}$ Lei Lia Wenjun Yang ${ }^{a}$ \\ Zhengping Zhuang ${ }^{\mathrm{b}}$ Jiang $\mathrm{Li}^{\mathrm{a}}$
}

aDepartment of Oral Pathology, Shanghai Ninth People's Hospital, College of Stomatology, Shanghai Jiao Tong University School of Medicine, Shanghai, National Clinical Research Center for Oral Diseases, Shanghai, Shanghai Key Laboratory of Stomatology \& Shanghai Research Institute of Stomatology, Shanghai, China, ${ }^{b}$ Neuro-Oncology Branch, National Cancer Institute, National Institutes of Health, Bethesda, MD, 'Duke University, Durham, NC, USA

\section{Key Words}

Mucoepidermoid carcinoma • Protein phosphatase 2A • LB100 • Chemotherapy • PI3K/AKT pathway

\begin{abstract}
Background/Aims: Protein phosphatase 2A (PP2A) is a ubiquitous serine/threonine phosphatase that mediates cell cycle regulation and metabolism. Mounting evidence has indicated that PP2A inhibition exhibits considerable anticancer potency in multiple types of human cancers. However, the efficacy of PP2A inhibition remains unexplored in mucoepidermoid carcinoma (MEC), especially in locally advanced and metastatic cases with limited systemic treatment. In this study, we demonstrated the therapeutic potency of LB100 in mucoepidermoid carcinoma. Methods: In this study, the expression of PP2A was evaluated using immunohistochemical (IHC) staining. The effects associated with LB100 alone and in combination with cisplatin for the treatment of mucoepidermoid carcinoma were investigated both in vitro, regarding metabolism, proliferation, and migration, and in vivo in a mucoepidermoid carcinoma xenograft model. In addition, with LB100 treatment and in response to an insulin stimulus, the expression levels and phosphorylation levels of targets in the PI3K-AKT pathway were determined using western blot analysis and immunoblotting. Results: The expression of protein phosphatase 2A was significantly upregulated in the clinical specimens of high-grade MECs compared with those of low-/medium-grade MECs and normal controls. In this article, we report that a small molecule PP2A inhibitor, LB100, decreased

L. Liu, H. Wang and J. Cui contributed equally to this study.

\begin{tabular}{ll}
\hline Zhengping Zhuang & Neuro-Oncology Branch, National Cancer Institute, National Institutes of Health, Bethesda, MD (USA); \\
and Jiang Li & Department of Oral Pathology, Shanghai Ninth People's Hospital, Shanghai Jiao Tong University \\
& School of Medicine, Shanghai (China); E-Mail zhengping.zhuang@nih.gov; lij1024@sh9hospital.org
\end{tabular}
\end{abstract}


cellular viability and glycolytic activity and induced G2/M cell cycle arrest. Importantly, LB100 enhanced the efficacy of cisplatin in mucoepidermoid carcinoma cells both in vitro and in vivo. PP2A inhibition by LB100 increased the phosphorylation of insulin receptor substrate 1(IRS1) on serine residues, downregulated the expression of phosphatidylinositol 3-kinase (PI3K) p110 alpha subunit and dephosphorylated AKT at Ser473 and Thr308 in mucoepidermoid carcinoma cells in response to insulin stimulus. Conclusion: These results highlight the translational potential of PP2A inhibition to synergize with cisplatin in mucoepidermoid carcinoma treatment.

(C) 2018 The Author(s)

Published by S. Karger AG, Basel

\section{Introduction}

Mucoepidermoid carcinoma (MEC) is the most common malignant salivary gland tumor, accounting for approximately $30 \%$ of all malignant tumors in the salivary glands $[1,2]$. Although most cases are low-/medium-grade lesions, $15.1 \%$ of cases are classified as highgrade lesions and are ineligible for systemic treatment, leading to a poor prognosis with a 5 -year survival rate of $47.2 \%[3,4]$. Surgery is the mainstay for the treatment of high-grade MEC. However, disfiguration and dysfunction ensue as complications of extensive radical parotidectomy. Moreover, adjuvant treatment has thus far demonstrated limited success in the treatment of high-grade MEC. While postoperative radiotherapy is generally advocated, approximately one-third of patients can still develop distant metastases [5]. Owing to the modest response of high-grade MEC to conventional chemotherapy, there is a major unmet need to develop targeted therapies.

Protein phosphatase 2A (PP2A) is a highly conserved serine/threonine phosphatase and is involved in a variety of important cellular processes, including migration, cell cycle progression, metabolism and apoptosis [6]. PP2A is considered to be a tumor suppressor due to its dephosphorylation activity [7]. However, PP2A has also been shown to promote carcinogenesis and tumor progression in several malignancies [8, 9]. Moreover, targeting PP2A has recently gained considerable momentum as an anticancer therapy. In a profusion of preclinical studies, PP2A inhibition by okadaic acid and cantharidin has been shown to have significant anti-tumor efficacy in pancreatic cancer [10], malignant testicular germ cell tumors [11], and leukemia [12,13]. However, okadaic acid and cantharidin are less likely to become mainstream treatment agents due to their severe toxic side effects [14]. LB100 is a novel small molecule inhibitor of PP2A and is currently being evaluated in phase I clinical trials, showing acceptable toxicity $[15,16]$. Strikingly, LB100 has shown promising efficacy as a chemo- and radio-sensitizer in a number of solid tumors, including glioblastoma [17], hepatocellular carcinoma [18], head and neck squamous cell carcinoma [19], osteosarcoma [20], pancreatic cancer [21], and medulloblastoma [22]. However, the effect of PP2A inhibition has not been explored in mucoepidermoid carcinoma (MEC), and none of the published literature to date has proposed to test the chemosensitizing effect of LB100 in immortalized cell lines stimulated with insulin.

PP2A inhibition has been reported to increase serine/threonine phosphorylation on insulin receptor substrate 1(IRS-1). In addition, phosphoserine residues in IRS-1 are involved in the negative regulation of the insulin signaling pathway and subsequent downstream tumorigenic signaling cascades, including the phosphatidylinositol 3-kinase/ AKT pathway [23]. It has been conclusively shown that the PI3K/AKT pathway regulates invasion, angiogenesis and metastasis in a series of cancer models [24, 25]. Therefore, we hypothesized that LB100 could sensitize treatment-refractory mucoepidermoid carcinoma to cisplatin by deactivating the IRS-1-mediated PI3K/AKT pathway.

In the present study, we performed experiments to explore the potential of LB100 in conjunction with cisplatin in the treatment of mucoepidermoid carcinoma. The effects of LB100 on insulin-stimulated IRS-1-mediated PI3K/AKT targets were measured to provide mechanistic information about MEC cells. 


\section{Cellular Physiology Cell Physiol Biochem 2018;50:317-331 \begin{tabular}{ll|l} 
and BiOChemistry & DOI: 10.1159/000494008 & $\begin{array}{l}\text { (C) } 2018 \text { The Author(s). Published by S. Karger AG, Basel } \\
\text { www.karger.com/cpb }\end{array}$
\end{tabular}}

Liu et al.: LB100 Sensitizes Mucoepidermoid Carcinoma to Chemotherapy

\section{Materials and Methods}

\section{Ethics Statement}

All animal studies in the present article conformed to internationally accepted standards and were performed in accordance with protocols approved by the Animal Care and Use Committee of the Ninth People's Hospital, Shanghai Jiaotong University School of Medicine.

\section{Clinical samples}

Tissues from 63 cases of human mucoepidermoid carcinoma and normal salivary gland tissues were collected from Shanghai Ninth People's Hospital, Shanghai Jiao Tong University School of Medicine. The histology and clinical stage were classified according to the WHO TNM staging system for salivary gland tumors. All the patients provided written informed consent before enrollment, and the study was approved by the Research Ethics Committee of Shanghai Ninth People's Hospital, Shanghai Jiao Tong University School of Medicine.

\section{Immunohistochemical staining}

After tissue sections were deparaffinized, rehydrated with a graded ethanol series, incubated with 0.1 mol/L sodium citrate (Sangon, Shanghai, China) for 20 minutes and blocked with $10 \%$ BSA (Sangon), the slides were incubated with anti-PP2A alpha+beta antibody (Abcam, Cambridge, UK at $4{ }^{\circ} \mathrm{C}$ overnight. After washing, the slides were labeled with an HRP-conjugated secondary antibody (Sangon) at room temperature for 1 hour. Positive staining was visualized with a DAB substrate solution (Sangon), and counterstaining was performed with hematoxylin. According to the ratio of positive-staining cells, 0-5\% scored 0, 6-35\% scored 1, 36-70\% scored 2 , and more than $70 \%$ scored 3 , and according to the staining intensity, no staining was scored as 0 , weak staining was 1 , moderate staining was 2 , and strong staining was 3 . The final score was determined using the score for the percent of positive cells $\times$ the staining intensity score as follows: "-" for a score of $0-1$; "+" for a score of 2-3; "++" for a score of 4-6; and "+++" for a score of $>6$. Low expression was defined as a total score $<4$, and high expression was defined as a total score $\geq 4$. These scores were determined independently by two senior pathologists in a blinded manner.

\section{Cells and reagents}

The human mucoepidermoid carcinoma cell lines UM-HMC 1, UM-HMC 3A and UM-HMC 3B were kindly provided by Dr. Jacques E. Nör (University of Michigan). Each cell line was tested and authenticated in their lab [26]. Cells were cultured in Dulbecco's Modified Eagle's Medium (Invitrogen, Carlsbad, CA, USA) supplemented with 10\% fetal bovine serum (Invitrogen), 1\% penicillin/streptomycin (Invitrogen), $20 \mathrm{ng} /$ $\mathrm{ml}$ epidermal growth factor (Sigma-Aldrich, St. Louis, MO, USA), $400 \mathrm{ng} / \mathrm{ml}$ hydrocortisone (Sigma-Aldrich), and $5 \mu \mathrm{g} / \mathrm{ml}$ insulin (Sigma-Aldrich). All cells were maintained in a humidified atmosphere of $5 \% \mathrm{CO}_{2}$ at $37^{\circ} \mathrm{C}$. The PPP2R2A expression vector HA-mPPP2R2A was established by inserting the Ppp2r2a cDNA (Han's lab, Xiamen University, China) into a linearized pCDNA4A-HA vector (HindIII and EcoR1). LB100 was provided by Lixte Biotechnology Holdings, Inc., dissolved in PBS to a stock concentration of $100 \mathrm{mM}$, and then stored at $-80^{\circ} \mathrm{C}$. For the in vivo and in vitro experiments, solutions were diluted from the stock solution immediately before administration.

\section{Cell viability assay}

Cell viability was assessed with a CCK8 assay kit (Dojindo, Japan). Approximately $4 \times 10^{3}$ UM-HMC 1 cells or $6 \times 10^{3}$ UM-HMC 3A and UM-HMC 3B cells in $100 \mu \mathrm{l}$ of medium were seeded into each well of 96well plates. After culturing overnight, the cells were treated with various concentrations of LB100 and/or cisplatin. To assess the ability of LB100 to enhance the cytotoxicity of cisplatin, the cells were pretreated with LB100 for 3 hours before the administration of cisplatin. After a 48-hour treatment, the supernatant of each well was removed, and CCK8 solution was added to give a final concentration of $1 \mathrm{mg} / \mathrm{ml}$ before the cells were incubated for another 2 hours. Absorbance values were determined at 450 nanometers with an ELx800 spectrophotometer (BioTek, Winooski, VT, USA). All the CCK8 assays were performed in triplicate. 


\section{Cellular Physiology Cell Physiol Biochem 2018;50:317-331

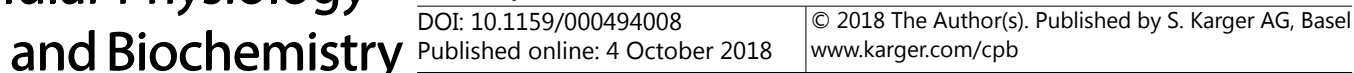

Liu et al.: LB100 Sensitizes Mucoepidermoid Carcinoma to Chemotherapy

PP2A activity assay

PP2A enzyme activity was assessed using the Ser/Thr Phosphatase Assay Kit 1 (Millipore, Billerica, MA, USA) per the manufacturer's instructions. Briefly, UM-HMC1 cells were cultured in a six-well plate. When the cells were $80 \%$ confluent, the medium was removed, and new media containing either a titration of LB100, cisplatin $(2 \mu \mathrm{g} / \mathrm{ml})$, or LB100 $(5 \mu \mathrm{M})$ plus cisplatin $(2 \mu \mathrm{g} / \mathrm{ml})$ were added. After four hours, the cells were washed with a $0.9 \%$ normal saline solution. Protein was extracted using a sonication protocol. Supernatants containing $100 \mu \mathrm{g}$ of total cellular protein were assessed using the PP2A Phosphatase Assay Kit according to the manufacturer's protocol. Experiments were performed in triplicate, and the data are presented as the mean percentage of relative PP2A activity compared with the control \pm SE.

\section{Migration assay}

Cells were seeded on the upper chamber of an 8- $\mu \mathrm{m}$-pore transwell insert (Costar, Tewksbury, MA, USA) at $1 \times 10^{5}$ cells/insert in DMEM and subjected to serum deprivation overnight. Three hundred microliters of complete DMEM with LB100 and/or cisplatin was then added to the lower chamber and incubated for 24 hours. Nonmigratory cells on the upper surface of the membrane were removed, and the cells that migrated through the membrane were fixed with $3.7 \%$ buffered formalin solution and stained with $0.5 \%$ crystal violet. The membranes were mounted on a microscope slide, and photographs of random fields were obtained using a light microscope and an AxioCam digital camera (Zeiss, Oberkochen, Germany). Each experiment was performed in triplicate.

\section{Cell cycle analysis}

Approximately $1 \times 10^{6}$ cells were plated per well in 6-well plates and treated with the indicated concentration of LB100 and/or cisplatin for 12 hours. EdU solution (Invitrogen) was added to the cell medium at a final concentration of $10 \mu \mathrm{M}$ for 1.5 hours using the Click-iT Plus EdU Alexa Fluor 647 Flow Cytometry Assay Kit (Invitrogen). After counterstaining with DAPI to assess the total DNA content per cell, the cells were analyzed by flow cytometry (LSRII, BD Biosciences, New Jersey, USA). The data analysis was performed using the FlowJo software (TreeStar).

\section{Glycolytic and mitochondrial respiration rate measurements}

A Seahorse XF96 instrument was used for the metabolic measurements. Cells were seeded at a density of $1 \times 10^{4}$ per well and incubated overnight. LB100 was then added to the medium, and the cells were incubated for 4 hours. The extracellular acidification rate (ECAR) and oxygen consumption rate (OCR) for each well were evaluated while the cells were subjected to the XF Cell Mito or the XF glycolytic stress test (Seahorse Bioscience).

\section{Western blot analysis}

Mucoepidermoid carcinoma cells were grown to $80 \%$ confluence in a $10-\mathrm{cm}$ plate and were treated with a titration of LB100 as indicated. There were also two groups of cells cultured with/without insulin and treated with $10 \mu \mathrm{M}$ LB100 and vehicle. After a 4-hour incubation, the cells were washed with ice-cold PBS and lysed in RIPA buffer (Thermo Scientific) supplemented with Complete Protease Inhibitor Cocktail tablets (Sigma-Aldrich) and sodium fluoride (Sigma-Aldrich), sodium orthovanadate (Sigma-Aldrich), sodium pyrophosphate (Sigma-Aldrich) and PMSF (Cell Signaling Technology, Danvers, MA, USA). Protein concentration was determined via a Bradford assay (BCATM, Pierce Biotechnology, Rockford, IL, USA). A total of $50 \mu \mathrm{g}$ of cell lysate was separated on a NuPage 4-12\% Bis-Tris gel (Invitrogen). Immune complexes were collected with LDS Sample Buffer (Invitrogen) and separated on a NuPage 4-12\% Bis-Tris gel (Invitrogen). Proteins were transferred onto polyvinylidene fluoride (PDVF) membranes (Invitrogen) using the iBlot2 dry blotting system (Invitrogen). The membranes were blocked with blocking buffer containing $5 \%$ nonfat dry milk in PBS with $0.1 \%$ Tween 20 and incubated overnight with primary antibodies against PI3K p110 alpha (Cell Signaling Technology), pan-AKT (Cell Signaling Technology), pAKT S473 (Cell Signaling Technology), and pAKT T308 (Abcam). The secondary antibodies, goat anti-mouse and goat antirabbit antibodies conjugated with horseradish peroxidase label (ThermoFisher), were diluted at 1:1000, and the membranes were incubated for 1 hour at room temperature. Finally, the immunoreactive bands were scanned and analyzed using a gel imaging system (Bio-Rad, Philadelphia, PA). GAPDH was used as an internal control. 


\section{Cellular Physiology Cell Physiol Biochem 2018;50:317-331

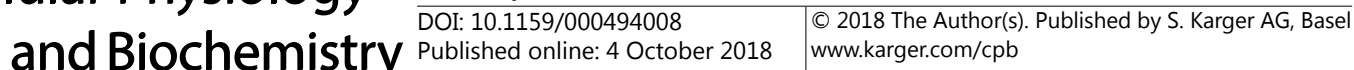

\section{Immunoprecipitation}

Cells in 10-cm plates were washed with ice-cold PBS and lysed in immunoprecipitation lysis buffer (Abcam) and 10\% NP-40 (ThermoFisher) with Complete Protease Inhibitor Cocktail tablets (Sigma-Aldrich). The lysates were precleared with protein G-agarose beads (Sigma-Aldrich) for 0.5 hours and then separately incubated with anti-IRS-1 antibody (Cell Signaling) and anti-HA antibody (BioLegend, San Diego, CA, USA) for 3 hours at $4{ }^{\circ} \mathrm{C}$, followed by the addition of protein G-agarose beads. Immunoblotting was performed as described in the western blot analysis methods.

\section{Subcutaneous tumorigenesis in NSG mice}

All animal studies were performed in accordance with protocols approved by the Animal Care and Use Committee of Ninth People's Hospital, Shanghai Jiaotong University, School of Medicine. Briefly, 6-8-weekold male NSG mice were subcutaneously injected with $5 \times 10^{6} \mathrm{UM}-\mathrm{HMC} 1$ cells in the left flank. All mice with a tumor size of approximately $100 \mathrm{~mm}^{3}$ were randomized into four groups (five animals in each group): saline (control), LB100 treatment, cisplatin treatment and combination treatment. LB100 and cisplatin were injected intraperitoneally (i.p.) at $1.5 \mathrm{mg} / \mathrm{kg}$ and $2.5 \mathrm{mg} / \mathrm{kg}$, respectively, every 4 days. Tumor size was monitored every 4 days according to the length $\times$ width $^{2} / 2$. The mice were sacrificed when the tumor size reached $1000 \mathrm{~mm}^{3}$, and xenografts were fixed with $3.7 \%$ buffered formalin solution for histological analysis.

\section{Statistical analysis}

The correlation of PP2A expression with clinicopathologic parameters in patients with MEC was evaluated using the chi-square test with the SPSS software program (version 17.0; IBM Corporation). Cell size was quantified using the Image-Pro Plus software (version 7.0, MEDIA CYBERNETICS). Western blot densitometry analysis was performed using ImageJ. Other graphical representations and statistical analyses were performed using GraphPad Prism 6. The results were analyzed using Student's t-test to compare the means between two groups and an ANOVA to compare the means among three or more groups. The data are expressed as the means \pm SEM. A value of $p<0.05$ was considered statistically significant.

\section{Results}

Correlations between PP2A expression and clinical parameters in patients with mucoepidermoid carcinoma

To illustrate the expression pattern of PP2A in mucoepidermoid carcinoma, immunohistochemicalanalysiswasperformed toidentifyPP2A expressioninmucoepidermoid carcinoma tissue and normal salivary gland tissue. We found that the expression of PP2A was pronouncedly elevated in high-grade mucoepidermoid carcinoma tissue $(n=21)$ compared with low-/medium-grade tumor tissue $(\mathrm{n}=26)$ and normal salivary gland tissue $(\mathrm{n}=15)$ (Fig. 1B). Representative staining of PP2A expression in normal salivary gland tissue, low-/medium-grade and high-grade mucoepidermoid carcinoma tissue is shown in Fig. 1A. To determine the clinical significance of PP2A expression in mucoepidermoid carcinoma, the chi-square test was used to assess the relationships between PP2A expression and the corresponding clinicopathologic parameters of the patient, including age, sex, and tumor location, size, T classification, lymph node metastasis, perineural invasion, histological grade and recurrence. The results showed that PP2A expression was significantly correlated with tumor location ( $p=0.033)$, tumor size $(p=0.049)$, T classification $(p=0.013)$ and histological grade $(p=0.002)$ (Table 1$)$. Taken together, the above data suggest that upregulated PP2A predicts a high histological grade and might contribute to tumor progression in mucoepidermoid carcinoma. 
Fig.1.PP2A expression was pronouncedly elevated in high-grade mucoepidermoid carcinoma tissues. (A) Representative photographs of hematoxylin and eosin (HE) staining and immunohistochemical staining of PP2A in normal salivary

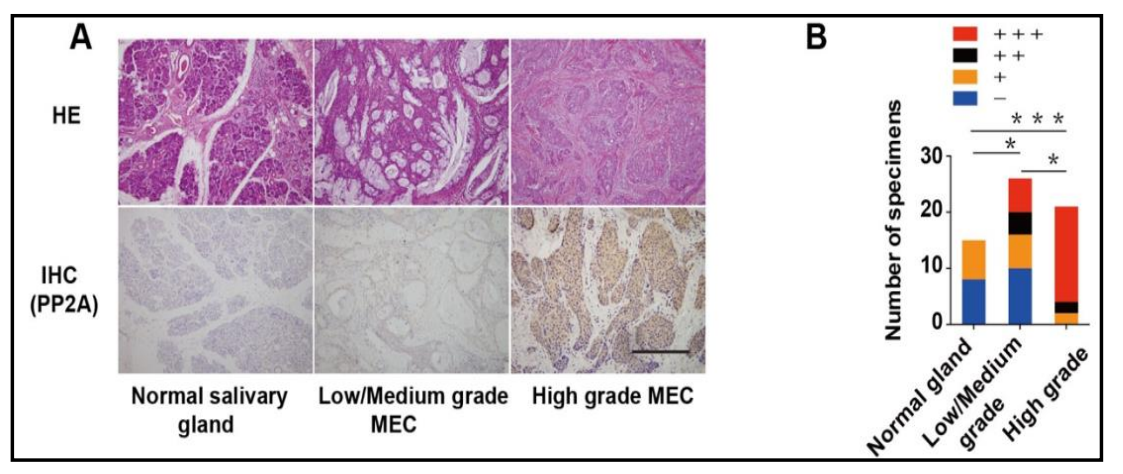
gland (left), low-/medium-grade MEC (middle) and high-grade MEC (right) tissues are shown. 400X. (B) Comparisons of PP2A expression by means of IHC analysis in normal salivary glands, low-/medium-grade MECs and high-grade MECs are shown. ${ }^{*} \mathrm{p}<0.05,{ }^{* *} \mathrm{p}<0.01,{ }^{* * *} \mathrm{p}<0.001$.

PP2A inhibition by LB100 decreased cell viability and glycolytic activity in mucoepidermoid carcinoma tumor cells in vitro

To assess the biological cellular changes that occur with PP2A inhibition in response to LB100 in mucoepidermoid carcinoma cells, we treated cells with LB100 for 48 hours and tested the cell viability of three different mucoepidermoid carcinoma cell lines: UM-HMC 1 , UMHMC $3 \mathrm{~A}$ and UM-HMC 3B. The CCK8 assay showed dose-dependent inhibition of cell growth in all the cell lines. The 50\% inhibitory concentration (IC50) of LB100 was $4.0,13.5$, and 14.8 $\mu$ M for UM-HMC1, UM-HMC 3A and UM-HMC 3B cells, respectively (Fig. 2A). Compared to the controls, cells exposed to relatively high LB100 concentrations underwent dramatic morphological changes, such as a decrease in cell size and an increased nucleus/plasma ratio (Fig. 2B).

Metabolic disorders and reprogramming could be one of the causes of chemo-sensitivity and can explain how PP2A plays an essential role in metabolism [27]. Therefore, we quantified the glycolytic activity and mitochondrial respiratory capacity using a Seahorse XF96 instrument (Fig. 2C). After incubating the cells with LB100 at $10 \mu \mathrm{M}$ for 4 hours, the UM-HMC1 and UM-HMC 3A cell lines both displayed significantly decreased glycolytic activity compared with the control groups $(p<0.05)$. Moreover, PP2A inhibition enhanced mitochondrial respiration in UM-HMC 1 and UM-HMC 3A cells; however, these differences were not statistically significant $(p>0.05)$. In summary, our findings suggest that PP2A plays an important role in metabolic reprogramming in MEC cells. 


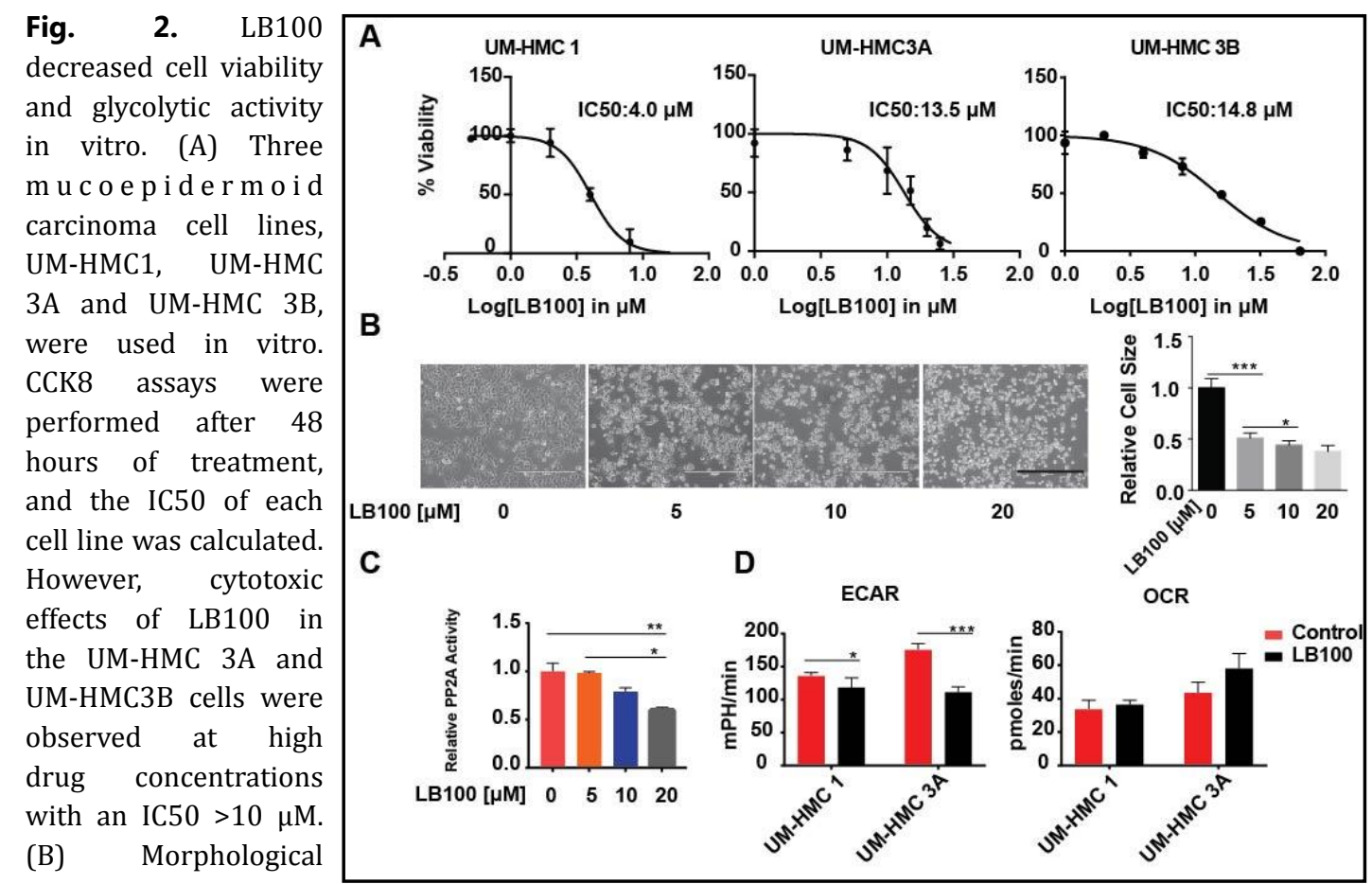

(B) Morphological

changes in UM-HMC1

cells treated with increasing LB100 concentrations for 48 hours are shown (left). Scale bar: $400 \mu \mathrm{m}$. Quantification and statistically significant differences compared with the control are shown (right). (C) Quantitative assessments of the extracellular acidification rate (ECAR, left) and oxygen-consumption rate (OCR, right) are shown. The data represent the means \pm SEM from two independent experiments (Student's $\mathrm{t}$-test was used to compare cultures with versus without LB100). ${ }^{*} \mathrm{p}<0.05,{ }^{* *} \mathrm{p}<0.01,{ }^{* * *} \mathrm{p}<0.001$.

\section{LB100 enhanced the effects of chemotherapy in MEC tumor cells in vitro}

We next examined whether PP2A inhibition by LB100 enhances the sensitivity of MEC tumor cells to cisplatin. The results of pretreating with LB100 showed a reduction in the IC50 from $2.848 \mu \mathrm{g} / \mathrm{ml}$ to $1.834 \mu \mathrm{g} / \mathrm{ml}$ for UM-HMC1 cells, from $3.791 \mu \mathrm{g} / \mathrm{ml}$ to $1.76 \mu \mathrm{g} /$ $\mathrm{ml}$ for UM-HMC3A cells, and from $2.795 \mu \mathrm{g} / \mathrm{ml}$ to $0.4692 \mu \mathrm{g} / \mathrm{ml}$ for UM-HMC3B cells (Fig. $3 A$ ). We then explored the effect of LB100 in combination with cisplatin on viability. The CCK8 assay showed that the viability of the MEC tumor cells was significantly reduced in the combination group compared with the single-treatment groups (Fig. 3B).

A preclinical study has shown that chemo-reagents such as doxorubicin induce an increase in PP2A activity [21]. Furthermore, in this study, we found that LB100 abrogated the cisplatin-induced augmentation of PP2A activity from $116.7 \%$ in the cisplatin-treated group to $84.98 \%$ in the combination group compared with the control ( $p=0.011$ ) (Fig. 3C).

Mucoepidermoid carcinoma cells are known to metastasize to local lymph nodes [28], and we used transwell assays to determine the function of LB100 in cell mobility and migration. Migrated cells were stained after treatment. Representative images were obtained at the end of each assay (Fig. 3D). The results suggested that LB100 combined with cisplatin profoundly impaired the migratory properties of MEC cells and suppressed their ability to invade and metastasize (Fig. 3E).

Preclinical observations have suggested a mechanism by which PP2A inhibition regulates the cell cycle in cancer cells and increases their vulnerability to the cytotoxicity of chemoreagents [17]. To test this hypothesis, flow cytometry was performed after the treatments to assess the cell distribution in each phase of the cell cycle. As shown in Fig. 3F and 3G, LB100 alone resulted in an increase in the G2/M phase compared with the control cells. Additionally, following treatment with the LB100 and cisplatin combination, the proportion 
Fig. 3. LB100 e $\mathrm{n} \mathrm{h}$ a $\mathrm{n}$ c e d the effects of che motherapy in vitro. (A) IC50 values of cisplatin were assessed with and without LB100 in mucoepidermoid carcinoma cell lines. LB100 (5 $\mu \mathrm{M})$ was added 3 hours prior to cisplatin. (B) CCK8 assays to test cell viability were performed in UM-HMC1, UM-HMC3A and UM-HMC3B cells after treatment. LB100 (5 $\mu \mathrm{M})$ was added 3 hours prior to cisplatin (2.5 $\mu \mathrm{g} / \mathrm{ml}$ ). (C) PP2A activity was assessed after the treatment.

(D)

Transwell assays were performed in mucoepidermoid carcinoma cell lines after treatments. Cells were treated as follows: control, LB100 (5 $\mu \mathrm{M})$, cisplatin $(2.5 \mu \mathrm{g} /$ $\mathrm{ml}$ ) and LB100 plus cisplatin at the same concentrations. Representative images in each treatment group were obtained at the end of the assays. (E) Analytical results

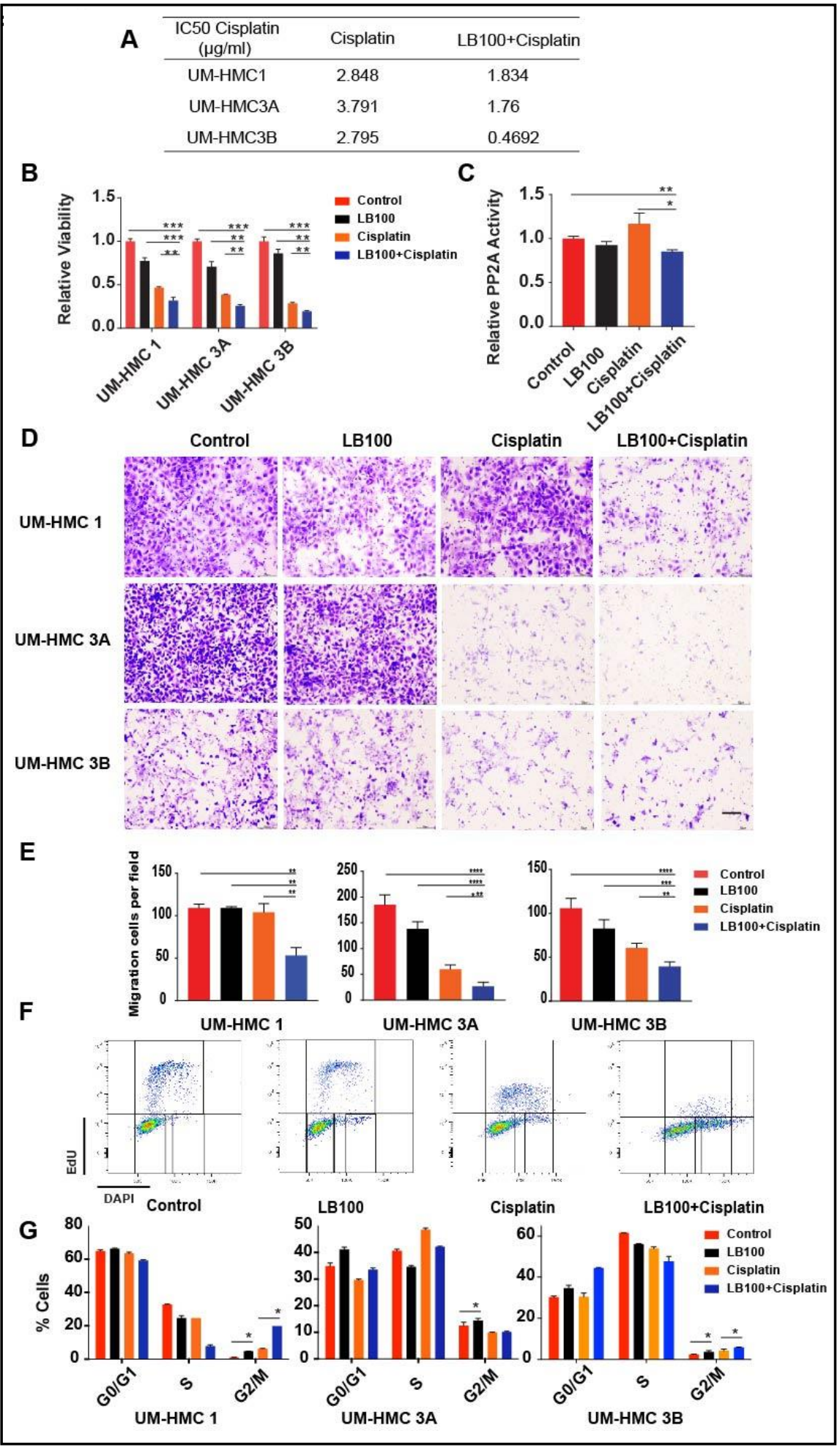
for the migrated

cells in each group are shown. (F) Cell cycle analysis via flow cytometry was performed to determine the relative percentages of UM-HMC1 and UM-HMC3A cells in the G0/G1, S and G2/M phases of the cell cycle using EDU and DAPI staining 12 hours after treatment. LB100 $(5 \mu \mathrm{M})$ was administered 3 hours prior to cisplatin $(2.5 \mu \mathrm{g} / \mathrm{ml})$. (G) Quantitative assessment of the cell percentage in each phase is shown. The data represent the means \pm SEM. ${ }^{*} \mathrm{p}<0.05,{ }^{* *} \mathrm{p}<0.01,{ }^{* * *} \mathrm{p}<0.001$. 
Liu et al.: LB100 Sensitizes Mucoepidermoid Carcinoma to Chemotherapy

of cells in the G2/M phase was also elevated compared with the single-treatment and control cells in all three mucoepidermoid carcinoma cell lines (Fig. 3G).

Taken altogether, these results suggest that PP2A inhibition by LB100 is a potential strategy for chemosensitization.

LB100 increased phosphoserine in IRS-1 and blocked the PI3K/AKT signaling pathway

Previous work has shown that PP2A inhibition can increase the serine/threonine phosphorylation of IRS-1, which modulates signal transduction in the insulin signaling pathway [29]. To test the effect of LB100 in the insulin-stimulated UM-HMC 1 cell line, immunoprecipitation with an anti-IRS-1 antibody followed by immunoblotting with an anti-phosphoserine antibody was performed. The results showed that LB100 increased phosphorylation at serine residues of IRS-1 in a dose-dependent manner after incubation with LB100 for 4 hours (Fig. 4A). To further confirm the mechanism involved in PP2A inhibition, targets in the PI3K/AKT pathway were also tested. The expression of PI3K p110 alpha was significantly reduced following treatment with $10 \mu \mathrm{M}$ and $20 \mu \mathrm{M}$ LB100. No significant change was observed in the expression of pan-AKT. However, there was a dose-dependent decrease in the phosphorylation of Ser473 and Thr308 residues in AKT (Fig. 4B). To further assess the inhibition of PP2A by LB100 upon insulin stimulation, cells were subjected to insulin deprivation overnight and then exposed to insulin, alone or in combination with LB100 $(10 \mu \mathrm{M})$. We found that the effect of insulin on AKT phosphorylation was partially compromised by PP2A inhibition. Similar results were obtained when assessing the protein level of PI3K p110 alpha (Fig. 4C). Similar results were also demonstrated in the UM-HMC3A cell line (Fig. 4F).

PP2A physiologically interacted with IRS-1 and the overexpression of PP2A decreased phosphoserine in IRS-1

To investigate the mechanism by which PP2A regulates IRS-1, we examined via coimmunoprecipitation whether PP2A could physically interact with IRS-1. UM-HMC1 cells were transfected with an expression vector containing PPP2R2A (HA-PPP2R2A), one PP2A regulatory subunit, or an empty vector. Cell lysates were then subjected to immunoprecipitation with an anti-HA-tag antibody followed by immunoblotting with an anti-IRS-1 antibody. The results suggested that PPP2R2A directly interacted with IRS-1 (Fig. 4D). In addition, the overexpression of PPP2R2A decreased phosphoserine in IRS-1 (Fig. 4E). A schematic of this proposed mechanism is presented in Fig. 4G.

LB100 enhanced the cytotoxicity effects of cisplatin in a mucoepidermoid carcinoma xenograft model

To confirm the chemosensitizing effect of LB100 in vivo, an MEC xenograft model was established by subcutaneously injecting UM-HMC1 cells into the right flank of 5-6-week-old NSG mice. The mice with a tumor burden (greater than $100 \mathrm{~mm}^{3}$ ) were randomized into four groups: control, LB100, cisplatin, and LB100 plus cisplatin. The treatment schedule is indicated in Fig. 5A. All the tumors grew slowly at first. However, at day 28, the combination of LB100 and cisplatin significantly decreased the tumor burden compared with the cisplatin-treated group $(p<0.05)$ (Fig. 5B). In the xenograft tumors, there were intermediate cells and mucin-producing cells, which displayed the classical histopathological patterns of mucoepidermoid carcinoma (Fig. 5C). In the control group, significant nodular invasion with perivascular involvement was observed (black arrow). In both the LB100 and the cisplatin monotherapy groups, the tumors were circumscribed with a relatively clear border, but these tumors exhibited remarkable invasion around the border. A clear infiltration of tumor cells into the muscle (blue circle) was observed in the cisplatin group. In the combination group, however, the tumors were well circumscribed with no invasion. Additionally, we observed significant necrosis in both the LB100 and the LB100 plus cisplatin groups (black circle) (Fig. 5D). 
Fig.4.LB100increased phosphoserine in IRS1 and deactivated the PI3K/AKT signaling pathway. After allowing the cells time to attach, increasing LB100 concentrations were added to each treatment group, and the cells were incubated for 4 hours. (A) The cells were lysed and subjected to immunoprecipitation (IP) with an anti-IRS-1 antibody followed by immunoblotting (IB) with an antibody against p hos phoserine. The western blot with densitometry analysis is shown. Statistically significant differences compared with the control are indicated by an asterisk $\left({ }^{*} \mathrm{p}<0.01\right)$. (B) Immunoblotting was used to analyze the expression of the PI3K regulatory subunit p110 $\alpha$, pan AKT and the phosphorylation of AKT residues at Ser473 and Thr308. The western blot with densitometry analysis is shown. (C) Cells were subjected to insulin deprivation overnight and then exposed to LB100
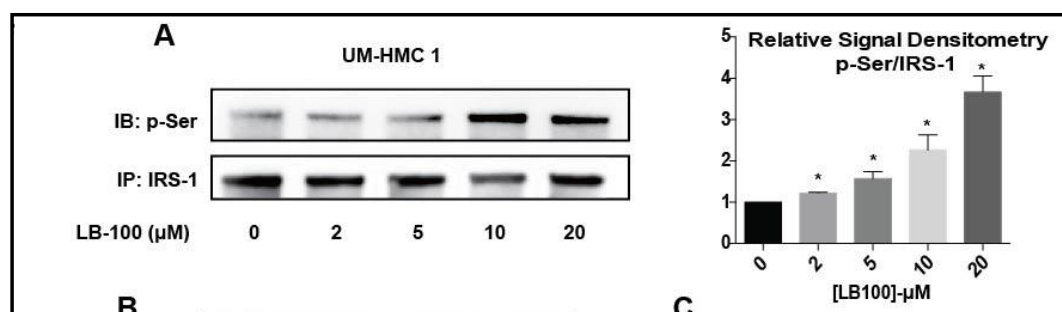

B
PI3K p110 alpha

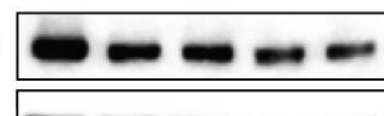
PI3K p110 alpha

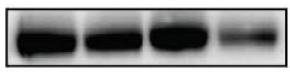

Pan AKT

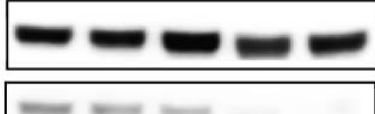

Pan AKT

PAKT S473

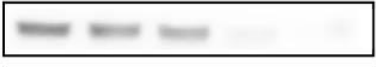

PAKT S473

PAKT T308

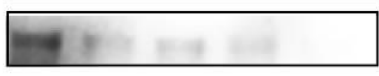

PAKT T308

GAPDH

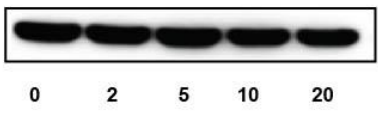

LB-100 ( $\mu \mathrm{M})$
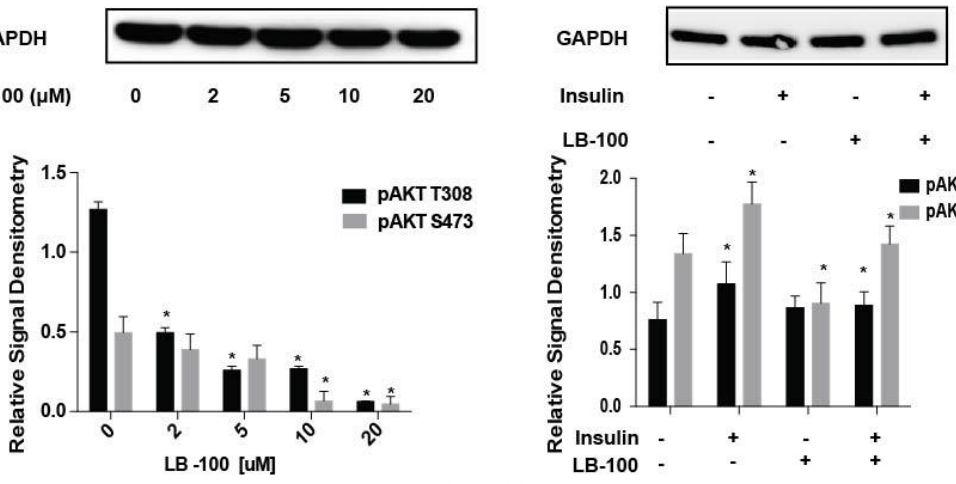

D
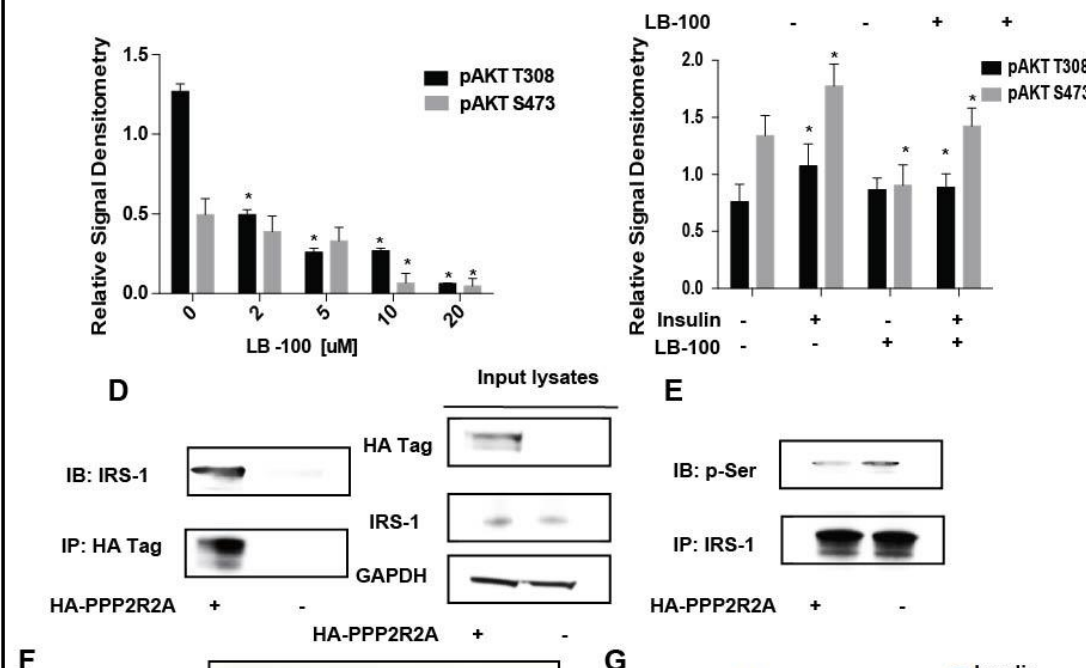

E

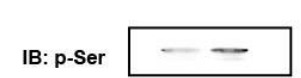

IP: IRS-1

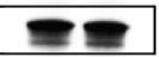

HA-PPP2R2A

G

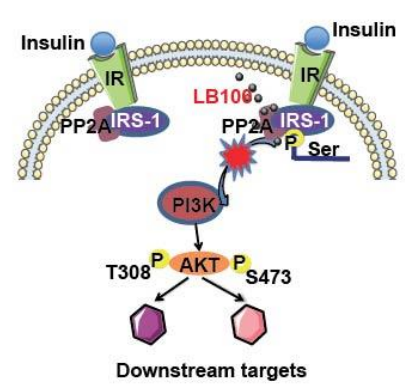

$(10 \mu \mathrm{M})$ alone, insulin alone or LB100 plus insulin for 4 hours and then analyzed by immunoblotting. The western blot with densitometry analysis is shown. PP2A physiologically interacted with IRS-1, and the overexpression of PP2A decreased phosphoserine in IRS-1. After transfection with an HA-tagged vector, the HA-PPP2R2A and UM-HMC1 cells were lysed. (D) Lysates were subjected to immunoprecipitation (IP) with an anti-HA-tag antibody followed by immunoblotting (IB) with an antibody against IRS-1. (E) Immunoprecipitates obtained with anti-IRS-1 antibodies were immunoblotted with antibodies directed against phosphoserine. (F) LB100 down-regulates the phosphorylation of AKT at Ser473 and Thr 308 in UM-HMC 3A cells. (G) A mechanistic illustration of the present study is shown. 
Fig. 5. LB100 enhanced the cytotoxicity of cisplatin in a xenograft model of mucoepidermoid carcinoma. (A) NSG mice were inoculated subcutaneously in the right flank with $5 \times 10^{6}$ UM-HMC1 cells. When the tumor reached $100 \mathrm{~mm}^{3}$, the mice were randomized to one of four treatment groups: control, LB100, cisplatin and combination. Treatments (LB100-1.5 $\mathrm{mg} / \mathrm{kg}$ and cisplatin-2.5 $\mathrm{mg} / \mathrm{kg}$ ) were administered every 4 days for 28 days. (B) Quantification of the tumor burden in the MEC xenograft model from post-treatment day 1 to day 28. The data are presented as the means+/SEM. (C) Representative HE staining of a classical mucoepidermoid carcinoma in the xenograft model, which shows mucinproducing (blackarrow) and intermediate cells (white arrow). (D) Histological features of tumors in NSG mice treated with vehicle, LB100, cisplatin and LB100 plus cisplatin. The control group showed significant nodular invasion with perivascular involvement (black arrow). The cisplatin-treated group showed the infiltration of tumor cells into the muscle (blue circle). The LB100 and the LB100 plus cisplatin-treated groups showed significant necrosis within a relatively well-circumscribed border (black circle). Scale bar: $60 \mu \mathrm{m}$.

\section{Discussion}

Cisplatin combination chemotherapy is a common practice in the treatment of a broad spectrum of refractory cancers. Efforts have been made to enhance the effectiveness of chemotherapy in mucoepidermoid carcinoma. However, the combination of cisplatin with mitomycin-C, adriamycin [30], paclitaxel [31], and vinorelbine [32] demonstrated only a modest response in clinical trials, albeit in a small series of patients. Therefore, novel therapeutic strategies are urgently needed to enhance the effectiveness of chemotherapy regimens against mucoepidermoid carcinoma. Exploiting molecularly specific pharmacologic interventions has achieved eradication in a broad range of cancers. Preclinical studies have shown that the inhibition of PP2A by a novel small molecule compound, LB100, enhances the antineoplastic activity of a variety of rationally designed anticancer agents via multiple mechanisms without significantly enhancing their toxicity [33]. To the best of our knowledge, this is the first report to demonstrate PP2A inhibition as a new therapeutic approach to enhancing the efficacy of cisplatin against mucoepidermoid carcinoma salivary gland tumors. 


\section{Cellular Physiology Cell Physiol Biochem 2018;50:317-331

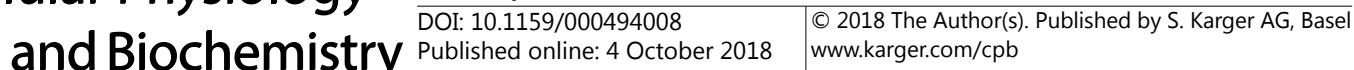

Liu et al.: LB100 Sensitizes Mucoepidermoid Carcinoma to Chemotherapy

It has been conclusively shown that PP2A inhibition in tumor cells leads to a downregulation of the DNA-damage response $[15,18,34]$ and the abrogation of cell cycle checkpoints to achieve preferential killing $[15,34,35]$. Consistent with those findings, we have shown that LB100 inhibited proliferation and induced a G2/M arrest in MEC cells.

The specificity and kinetics of the PP2A inhibitor LB100 regarding the inhibition of the Ser/Thr phosphatase activity of PP2A were determined in a recent report using the catalytic subunit of recombinant human PP2A and a phosphopeptide (K-R-pT-I-R-R) as a substrate [36]. Compared with multiple kinases, relatively few protein serine/threonine phosphatases (PSPs) control the specific dephosphorylation of thousands of phosphoprotein substrates. Thus, the mild inhibition of PP2A activity by LB100 even at $5 \mu \mathrm{M}$ caused severe inhibition and altered cell viability/proliferation in the present work.

A previous study has demonstrated that PP2A inhibition also re-established the metabolic profile of Treg cells in PP2A flox mice [37]. These authors showed that the transgenic knockdown of PP2A resulted in augmented glycolytic activity and mitochondrial respiratory capacity in the PP2A flox Tregs. However, in the MEC cell lines, the pharmacologic inhibition of PP2A with LB100 decreased the glycolytic rate and increased the oxidative phosphorylation rate. These discrepancies may be ascribed to the in vitro environment in this study, which involved an insulin stimulus and may have influenced the pharmacokinetics of LB100 and the cellular response. We also observed that LB100 alone showed limited utility with an IC50 $>10 \mu \mathrm{M}$ in 2 out of 3 MEC cell lines. A plasma concentration of LB100 $>10 \mu \mathrm{M}$ was unachievable, even with the acceptable toxicity dosage of $1.5 \mathrm{mg} / \mathrm{kg}$ in vivo, according to the pharmacokinetics study [34].

We further confirmed the effectiveness of LB100 in combination with cisplatin against mucoepidermoid carcinoma both in vitro and in vivo. This concept is based on the hypothesis that the selective killing of cancer cells by nonspecific cytotoxic drugs is achieved through the use of one drug at a dose that is sufficient to induce growth arrest but not cell death in the normal cell population, followed by a second drug that is incapable of fatally damaging the normal cells but is capable of killing the cancer cell population remaining in the cell cycle due to defects in checkpoint control [38]. Therefore, a combination treatment of LB100 and cisplatin could be a promising strategy against mucoepidermoid carcinoma. Consistent with earlier reports in other cancers, we demonstrated that PP2A inhibition in combination with cisplatin resulted in cell growth arrest, the deactivation of the G2/M checkpoint and the suppression of the motility and migration of MEC cells.

The activated PI3K/AKT pathway has been implicated in the process of tumorigenesis and malignant transformation [39]. Hyperphosphorylated AKT has been reported in many different types of salivary gland tumors, including mucoepidermoid carcinoma [40, 41]. Therefore, we hypothesized that the dephosphorylation of AKT could be another mechanism of LB100 action. Our experimental findings confirmed that PP2A inhibition by LB100 decreased the phosphorylation of AKT at Thr308 and Ser473, indicating deactivated AKT signaling. However, in certain cancer cell lines, PP2A inhibition can also result in enhanced AKT phosphorylation $[19,21]$. As a serine/threonine phosphatase, PP2A is known to dephosphorylate the phosphorylation sites of proteins. The most likely explanation for this discrepancy is that upstream targets of AKT are prioritized for phosphorylation in response to insulin. We demonstrated that PP2A inhibition by LB100 increased IRS-1 serine phosphorylation in response to an insulin stimulus. Interestingly, we also showed a decrease in the expression of PI3K p110 $\alpha$. A prior study has demonstrated that the absence of PI3K p110 $\alpha$ results in a dramatic decrease in insulin-stimulated PI3K activity and attenuates the phosphorylation of AKT at Thr308 and Ser473[42]. However, the mechanism by which PP2A inhibition decreases PI3K p $110 \alpha$ expression is not completely understood. Elevated IRS-1 serine phosphorylation and reduced PI3Kactivity are all mechanisms that lead to a reduction in AKT phosphorylation. In summary, our findings extend the current understanding of the molecular mechanisms that determine the vulnerability of MEC cells to PP2A inhibition through compromised AKT-associated phosphorylation. 


\section{Cellular Physiology Cell Physiol Biochem 2018;50:317-331 and Biochemistry \begin{tabular}{l|l} 
DOI: 10.1159/000494008 & (c) 2018 The Author(s). Published by S. Karger AG, Basel \\
www.karger.com/cpb
\end{tabular}

Liu et al.: LB100 Sensitizes Mucoepidermoid Carcinoma to Chemotherapy

\section{Conclusion}

The findings presented herein provide convincing proof-of-concept evidence that a pharmacological inhibitor of PP2A shows promising translational potential and achieves better cytotoxic effects when combined with cisplatin for the treatment of mucoepidermoid carcinoma, at least in part by suppressing the PI3K/AKT pathway. This treatment approach warrants further investigation in prospective clinical trials.

\section{Acknowledgements}

We extend special thanks to Dr. Jacques E. Nör (University of Michigan) for providing the UM-HMC 1, UM-HMC3A and UM-HMC3B mucoepidermoid carcinoma cell lines. We thank Ms. Shannon Skarshaug for helping us with the manuscript revisions. We also acknowledge Dr. F. Livak and K. Wolcott for technical support in the flow cytometry analysis.

Limin Liu, Herui Wang, Jing Cui, and Qi Zhang carried out all experiments and data analyses. Limin Liu, Wei Zhang, Wanlin Xu, Hao Lu, Shengwen Liu, Shukun Shen, Francia Fang, Lei $\mathrm{Li}$, and Wenjun Yang wrote the manuscript. Zhengping Zhuang and Jiang Li designed all the experiments.

This work was supported by the Intramural Research Program at the National Cancer Institute of the NIH, Shanghai Science and Technology Commission Program (No. 1614092200) and a Shanghai Municipal Education Commission-Gaofeng Clinical Medicine Grant.

\section{Disclosure Statement}

The authors declare they have no conflicts of interest.

\section{References}

1 Fonseca FP, Carvalho Mde V, de Almeida OP, Rangel AL, Takizawa MC, Bueno AG, Vargas PA: Clinicopathologic analysis of 493 cases of salivary gland tumors in a Southern Brazilian population. Oral Surg Oral Med Oral Pathol Oral Radiol 2012;114:230-239.

-2 Vargas PA, Gerhard R, Araujo Filho VJ, de Castro IV: Salivary gland tumors in a Brazilian population: a retrospective study of 124 cases. Rev Hosp Clin Fac Med Sao Paulo 2002;57:271-276.

3 Liu S, Ruan M, Li S, Wang L, Yang W: Increased expression of MUC1 predicts poor survival in salivary gland mucoepidermoid carcinoma. J Craniomaxillofac Surg 2014;42:1891-1896.

4 Clode AL, Fonseca I, Santos JR, Soares J: Mucoepidermoid carcinoma of the salivary glands: a reappraisal of the influence of tumor differentiation on prognosis. J Surg Oncol 1991;46:100-106.

-5 Terhaard CH, Lubsen H, Rasch CR, Levendag PC, Kaanders HH, Tjho-Heslinga RE, van Den Ende PL, Burlage F, Dutch H, Neck Oncology Cooperative G: The role of radiotherapy in the treatment of malignant salivary gland tumors. Int J Radiat Oncol Biol Phys 2005;61:103-111.

6 Perrotti D, Neviani P: Protein phosphatase 2A: a target for anticancer therapy. Lancet Oncol 2013;14:e229238.

7 Mumby M: PP2A: unveiling a reluctant tumor suppressor. Cell 2007;130:21-24.

8 Duong FH, Dill MT, Matter MS, Makowska Z, Calabrese D, Dietsche T, Ketterer S, Terracciano L, Heim MH: Protein phosphatase 2A promotes hepatocellular carcinogenesis in the diethylnitrosamine mouse model through inhibition of p53. Carcinogenesis 2014;35:114-122.

-9 Duong FH, Filipowicz M, Tripodi M, La Monica N, Heim MH: Hepatitis C virus inhibits interferon signaling through up-regulation of protein phosphatase 2A. Gastroenterology 2004;126:263-277. 


\section{Cellular Physiology Cell Physiol Biochem 2018;50:317-331 \begin{tabular}{l|l} 
and Biochemistry & DOI: 10.1159/000494008 \\
Publismed 2018 The Author(s). Published by S. Karger AG, Basel \\
www.karger.com/cpb
\end{tabular}

10 Li W, Xie L, Chen Z, Zhu Y, Sun Y, Miao Y, Xu Z, Han X: Cantharidin, a potent and selective PP2A inhibitor, induces an oxidative stress-independent growth inhibition of pancreatic cancer cells through G2/M cellcycle arrest and apoptosis. Cancer Sci 2010;101:1226-1233.

-11 Schweyer S, Bachem A, Bremmer F, Steinfelder HJ, Soruri A, Wagner W, Pottek T, Thelen P, Hopker WW, Radzun HJ, Fayyazi A: Expression and function of protein phosphatase PP2A in malignant testicular germ cell tumours. J Pathol 2007;213:72-81.

12 Riordan FA, Foroni L, Hoffbrand AV, Mehta AB, Wickremasinghe RG: Okadaic acid-induced apoptosis of HL60 leukemia cells is preceded by destabilization of bcl-2 mRNA and downregulation of bcl-2 protein. FEBS Lett 1998;435:195-198.

$\checkmark 13$ Boudreau RT, Conrad DM, Hoskin DW: Apoptosis induced by protein phosphatase 2A (PP2A) inhibition in T leukemia cells is negatively regulated by PP2A-associated p38 mitogen-activated protein kinase. Cell Signal 2007;19:139-151.

14 Bonness K, Aragon IV, Rutland B, Ofori-Acquah S, Dean NM, Honkanen RE: Cantharidin-induced mitotic arrest is associated with the formation of aberrant mitotic spindles and lagging chromosomes resulting, in part, from the suppression of PP2Aalpha. Mol Cancer Ther 2006;5:2727-2736.

15 Lu J, Kovach JS, Johnson F, Chiang J, Hodes R, Lonser R, Zhuang Z: Inhibition of serine/threonine phosphatase PP2A enhances cancer chemotherapy by blocking DNA damage induced defense mechanisms. Proc Natl Acad Sci U S A 2009;106:11697-11702.

-16 Chung V, Mansfield AS, Braiteh F, Richards D, Durivage H, Ungerleider RS, Johnson F, Kovach JS: Safety, Tolerability, and Preliminary Activity of LB-100, an Inhibitor of Protein Phosphatase 2A, in Patients with Relapsed Solid Tumors: An Open-Label, Dose Escalation, First-in-Human, Phase I Trial. Clin Cancer Res 2017;23:3277-3284.

17 Lv P, Wang Y, Ma J, Wang Z, Li JL, Hong CS, Zhuang Z, Zeng YX: Inhibition of protein phosphatase 2A with a small molecule LB100 radiosensitizes nasopharyngeal carcinoma xenografts by inducing mitotic catastrophe and blocking DNA damage repair. Oncotarget 2014;5:7512-7524.

18 Chang KE, Wei BR, Madigan JP, Hall MD, Simpson RM, Zhuang Z, Gottesman MM: The protein phosphatase 2A inhibitor LB100 sensitizes ovarian carcinoma cells to cisplatin-mediated cytotoxicity. Mol Cancer Ther 2015;14:90-100.

19 Zhu DW, Yuan YX, Qiao JK, Yu C, Yang X, Wang LZ, Zhang ZY, Zhong LP: Enhanced anticancer activity of a protein phosphatase $2 \mathrm{~A}$ inhibitor on chemotherapy and radiation in head and neck squamous cell carcinoma. Cancer Lett 2015;356:773-780.

20 Zhang C, Hong CS, Hu X, Yang C, Wang H, Zhu D, Moon S, Dmitriev P, Lu J, Chiang J, Zhuang Z, Zhou Y: Inhibition of protein phosphatase $2 \mathrm{~A}$ with the small molecule LB100 overcomes cell cycle arrest in osteosarcoma after cisplatin treatment. Cell Cycle 2015;14:2100-2108.

21 Bai X, Zhi X, Zhang Q, Liang F, Chen W, Liang C, Hu Q, Sun X, Zhuang Z, Liang T: Inhibition of protein phosphatase $2 \mathrm{~A}$ sensitizes pancreatic cancer to chemotherapy by increasing drug perfusion via HIF1alpha-VEGF mediated angiogenesis. Cancer Lett 2014;355:281-287.

-22 Ho WS, Feldman MJ, Maric D, Amable L, Hall MD, Feldman GM, Ray-Chaudhury A, Lizak MJ, Vera JC, Robison RA, Zhuang Z, Heiss JD: PP2A inhibition with LB100 enhances cisplatin cytotoxicity and overcomes cisplatin resistance in medulloblastoma cells. Oncotarget 2016;7:12447-12463.

23 Mothe I, Van Obberghen E: Phosphorylation of insulin receptor substrate-1 on multiple serine residues, 612, 632, 662, and 731, modulates insulin action. J Biol Chem 1996;271:11222-11227.

24 Ahmad A, Biersack B, Li Y, Kong D, Bao B, Schobert R, Padhye SB, Sarkar FH: Targeted regulation of PI3K/ Akt/mTOR/NF-kappaB signaling by indole compounds and their derivatives: mechanistic details and biological implications for cancer therapy. Anticancer Agents Med Chem 2013;13:1002-1013.

25 Luo J, Manning BD, Cantley LC: Targeting the PI3K-Akt pathway in human cancer: rationale and promise. Cancer Cell 2003;4:257-262.

26 Warner KA, Adams A, Bernardi L, Nor C, Finkel KA, Zhang Z, McLean SA, Helman J, Wolf GT, Divi V, Queimado L, Kaye FJ, Castilho RM, Nor JE: Characterization of tumorigenic cell lines from the recurrence and lymph node metastasis of a human salivary mucoepidermoid carcinoma. Oral Oncol 2013;49:10591066.

-27 Pinto BS, Orr-Weaver TL: Drosophila protein phosphatases 2A B' Wdb and Wrd regulate meiotic centromere localization and function of the MEI-S332 Shugoshin. Proc Natl Acad Sci U S A 2017;114:12988-12993. 


\section{Cellular Physiology Cell Physiol Biochem 2018;50:317-331 \begin{tabular}{ll|l} 
DOI: 10.1159/000494008 & C 2018 The Author(s). Published by S. Karger AG, Basel \\
www.karger.com/cpb
\end{tabular}

-28 Brandwein MS, Ivanov K, Wallace DI, Hille JJ, Wang B, Fahmy A, Bodian C, Urken ML, Gnepp DR, Huvos A, Lumerman H, Mills SE: Mucoepidermoid carcinoma: a clinicopathologic study of 80 patients with special reference to histological grading. Am J Surg Pathol 2001;25:835-845.

29 Ugi S, Imamura T, Maegawa H, Egawa K, Yoshizaki T, Shi K, Obata T, Ebina Y, Kashiwagi A, Olefsky JM: Protein phosphatase 2A negatively regulates insulin's metabolic signaling pathway by inhibiting Akt (protein kinase B) activity in 3T3-L1 adipocytes. Mol Cell Biol 2004;24:8778-8789.

-30 Creagan ET, Woods JE, Rubin J, Schaid DJ: Cisplatin-based chemotherapy for neoplasms arising from salivary glands and contiguous structures in the head and neck. Cancer 1988;62:2313-2319.

-31 Airoldi M, Fornari G, Pedani F, Marchionatti S, Gabriele P, Succo G, Bumma C: Paclitaxel and carboplatin for recurrent salivary gland malignancies. Anticancer Res 2000;20:3781-3783.

-32 Airoldi M, Pedani F, Succo G, Gabriele AM, Ragona R, Marchionatti S, Bumma C: Phase II randomized trial comparing vinorelbine versus vinorelbine plus cisplatin in patients with recurrent salivary gland malignancies. Cancer 2001;91:541-547.

33 Hong CS, Ho W, Zhang C, Yang C, Elder JB, Zhuang Z: LB100, a small molecule inhibitor of PP2A with potent chemo- and radio-sensitizing potential. Cancer Biol Ther 2015;16:821-833.

34 Ho WS, Sizdahkhani S, Hao S, Song H, Seldomridge A, Tandle A, Maric D, Kramp T, Lu R, Heiss JD, Camphausen K, Gilbert MR, Zhuang Z, Park DM: LB-100, a novel Protein Phosphatase 2A (PP2A) inhibitor, sensitizes malignant meningioma cells to the therapeutic effects of radiation. Cancer Lett 2018;415:217226.

35 Gordon IK, Lu J, Graves CA, Huntoon K, Frerich JM, Hanson RH, Wang X, Hong CS, Ho W, Feldman MJ, Ikejiri B, Bisht K, Chen XS, Tandle A, Yang C, Arscott WT, Ye D, Heiss JD, Lonser RR, Camphausen K, Zhuang Z: Protein Phosphatase 2A Inhibition with LB100 Enhances Radiation-Induced Mitotic Catastrophe and Tumor Growth Delay in Glioblastoma. Mol Cancer Ther 2015;14:1540-1547.

36 Lai D, Chen M, Su J, Liu X, Rothe K, Hu K, Forrest DL, Eaves CJ, Morin GB, Jiang X: PP2A inhibition sensitizes cancer stem cells to ABL tyrosine kinase inhibitors in BCR-ABL(+) human leukemia. Sci Transl Med DOI: 10.1126/scitranslmed.aan8735.

-37 Apostolidis SA, Rodriguez-Rodriguez N, Suarez-Fueyo A, Dioufa N, Ozcan E, Crispin JC, Tsokos MG, Tsokos GC: Phosphatase PP2A is requisite for the function of regulatory T cells. Nat Immunol 2016;17:556-564.

-38 Zhuang Z, Lu J, Lonser R, Kovach JS: Enhancement of cancer chemotherapy by simultaneously altering cell cycle progression and DNA-damage defenses through global modification of the serine/threonine phosphoproteome. Cell Cycle 2009;8:3303-3306.

39 Nicholson KM, Anderson NG: The protein kinase B/Akt signalling pathway in human malignancy. Cell Signal 2002;14:381-395.

40 Marques YM, de Lima Mde D, de Melo Alves Sde M, Jr., Soares FA, de Araujo VC, Pinto Ddos S, Jr., Mantesso A: Mdm2, p53, p21 and pAKT protein pathways in benign neoplasms of the salivary gland. Oral Oncol 2008;44:903-908.

41 Alamri AM, Liu X, Blancato JK, Haddad BR, Wang W, Zhong X, Choudhary S, Krawczyk E, Kallakury BV, Davidson BJ, Furth PA: Expanding primary cells from mucoepidermoid and other salivary gland neoplasms for genetic and chemosensitivity testing. Dis Model Mech DOI: 10.1242/dmm.031716.

-42 Nelson VL, Jiang YP, Dickman KG, Ballou LM, Lin RZ: Adipose tissue insulin resistance due to loss of PI3K p110alpha leads to decreased energy expenditure and obesity. Am J Physiol Endocrinol Metab 2014;306:E1205-1216. 